\title{
Importance of level mixing on accurate [Fe II] transition rates (Research Note)
}

\author{
N. C. Deb and A. Hibbert \\ Applied Mathematics and Theoretical Physics Department, Queen's University Belfast, Belfast BT7 1NN, Northern Ireland, UK \\ e-mail: a.hibbert@qub.ac.uk
}

Received 19 July 2010 / Accepted 13 September 2010

\section{ABSTRACT}

\begin{abstract}
Context. In a very recent measurement Gurell et al. $\left(2009\right.$, A\&A, 508, 525) commented that while the theoretical lifetime of $a^{4} \mathrm{G}_{5.5}$ is approximately one tenth of the lifetime of $b^{2} \mathrm{H}_{5.5}$ the corresponding measurement shows this to be close to one fifth. This large discrepancy is attributed to the effect of inadequate level mixing in the theoretical calculations.

Aims. The aim of this work is to make a detailed analysis of these level mixing effects on transitions from various lower levels to the $a^{4} \mathrm{G}_{5.5}$ and $b^{2} \mathrm{H}_{5.5}$ levels given in three previous calculations and in the present more extensive $\mathrm{CI}$ calculation.

Methods. The CIV3 structure codes of Hibbert (1975, Comput. Phys. Commun., 9, 141) and Hibbert et al. (1991, Comput. Phys. Commun., 64, 455) are used in the present work, combined with our "fine-tuning" extrapolation process.

Results. The calculated mixing between the upper levels, obtained in previous calculations, is shown to be too weak. The stronger mixing determined in our work gives rise to a calculated lifetime for $b^{2} \mathrm{H}_{5.5}$ within $3 \%$ of the measured value. On the other hand our calculated lifetime for $a^{4} \mathrm{G}_{5.5}$ is around $20 \%$ lower than the measured value, which has fairly wide error bars.

Conclusions. Our enhanced calculations explain the difference between previous calculations of the $b^{2} \mathrm{H}_{5.5}$ lifetime and the recent measured value and confirm the latter. We also suggest a somewhat higher value than experiment for the lifetime of $a^{4} \mathrm{G}_{5.5}$.
\end{abstract}

Key words. atomic data - atomic processes - methods: numerical - stars: individual: Eta Carinae

\section{Introduction}

Eta Carinae is a particularly rich source of forbidden [Fe II] emission lines, but they are also observed in the spectra of many other stellar objects. A detailed study of these spectra requires accurate atomic data, and particularly transition rates. The transitions discussed in this Note have been observed in $\eta$ Car (Gull et al. 1999), though they were first identified by Merrill (1928).

Using a laser probing technique (LPT) Gurell et al. (2009) recently measured the lifetimes of two metastable states of Fe II, namely those of $a^{4} \mathrm{G}_{5.5}$ and $b^{4} \mathrm{D}_{0.5}$ belonging to $3 \mathrm{~d}^{6}\left({ }^{3} \mathrm{G}\right) 4 \mathrm{~s}$ and $3 d^{6}\left({ }^{3} D\right) 4 s$ configurations respectively. They also concluded that lifetime of $b^{2} \mathrm{H}_{5.5}$ measured previously by Hartman et al. (2003) was found to be only five times greater than that of its neighbour $a^{4} \mathrm{G}_{5.5}$ whereas in earlier theoretical results this factor ranged from 7.4 (Quinet et al. 1996) through 11.9 (Garstang 1962). In the present research note we explain this discrepancy in terms of the level mixing of the two neighbouring levels separated by $741 \mathrm{~cm}^{-1}$. We also show, through our large scale CI calculation, that the apparent agreement of lifetimes of $a^{4} \mathrm{G}_{5.5}$ between the new measurement (Gurell et al. 2009) and previous calculations may be better than the accuracy of either result.

Regarding the experimental method, it was demonstrated by Palmeri et al. (2008) that at least some LPT measurements contained errors as large as $50 \%$ or more, even without the experimental uncertainties. This was shown (Palmeri et al. 2008) for the lifetimes measurements of $3 \mathrm{~d}^{2}\left({ }^{3} \mathrm{P}\right) 4 \mathrm{~s} b^{4} \mathrm{P}_{2.5}$ and $3 \mathrm{~d}^{2}\left({ }^{3} \mathrm{P}\right) 4 \mathrm{~s}$ $b^{2} \mathrm{P}_{0.5}$ levels of Ti II. For both these levels the calculated lifetimes are shown to be closer to the corrected measurement of Palmeri et al. (2008) based on an innovative idea of Royen et al. (2007) which avoids the repopulation effects. Additionally, it is also well known that within the storage ring, particle collisions sometimes impact negatively on the measured lifetimes: collisional de-excitation leads to quenching, yielding lifetimes that appeared shorter than pure radiative lifetimes.

\section{Theoretical method}

In the present work we have used the CIV3 code (Hibbert 1975; Hibbert et al. 1991) to compute M 1 and E 2 [Fe II] transitions between all levels of $3 d^{7}, 3 d^{6} 4 s$ and $3 d^{5} 4 s^{2}$ configurations. Here we report on a few of them which have attracted observational and laboratory interest. We have used almost the same set of optimised orbitals generated in recent calculations from our group (Corrégé \& Hibbert 2005, 2006). However, we have made one change: in order to get better ab initio energy differences for the $3 \mathrm{~d}^{7}$ levels we used the Hartree-Fock (HF) $3 \mathrm{~d}$ function given by Clementi and Roetti (1974) for $3 d^{7}{ }^{4} \mathrm{~F}$ instead of their $3 \mathrm{~d}$ of the $3 d^{6} 4$ s ground configuration chosen in previous calculations (Corrégé \& Hibbert 2005, 2006).

The choice of configuration state functions (CSFs) is made in two steps: (a) initially the $3 \mathrm{~s}$ and $3 \mathrm{p}$ subshells are kept closed and up to two promotions are allowed from the valence subshells to $4 \mathrm{~s}, 4 \mathrm{p}, 4 \mathrm{~d}, 4 \mathrm{f}, 5 \mathrm{~s}, 5 \mathrm{p}, 5 \mathrm{~d}, 6 \mathrm{~s}, 6 \mathrm{p}, 6 \mathrm{~d}, 7 \mathrm{~s}$ and $7 \mathrm{p}$ orbitals, with all possible angular momentum couplings. LS energies of all the states of interest belonging to all possible symmetries are calculated. It is found that many of these CSFs produce negligibly small components (with an absolute value smaller than 0.001) in all the relevant eigenvectors of a given LS symmetry. We therefore deleted them for subsequent calculations; (b) we then allowed selective one and two promotions from $3 \mathrm{~s}$ and $3 \mathrm{p}$ subshells, just to $3 \mathrm{~d}$ and $4 l$ subshells. This introduces 15 core replacement 
configurations: $3 s 3 p^{6} 3 d^{8}, \quad 3 p^{6} 3 d^{9}, 3 s 3 p^{6} 3 d^{7} 4 s, 3 s 3 p^{6} 3 d^{7} 4 d$, $3 p^{6} 3 d^{7} 4 s^{2}, 3 p^{6} 3 d^{8} 4 s, 3 s^{2} 3 p^{4} 3 d^{9}, 3 s^{2} 3 p^{4} 3 d^{7} 4 s^{2}, 3 s^{2} 3 p^{4} 3 d^{8} 4 s$, $3 s^{2} 3 p^{4} 3 d^{7} 4 p^{2}, \quad 3 s^{2} 3 p^{5} 3 d^{7} 4 f, 3 s^{2} 3 p^{4} 3 d^{6} 4 s^{4} p^{2}, 3 s^{2} 3 p^{5} 3 d^{6} 4 s 4 f$, $3 s^{2} 3 p^{4} 3 d^{5} 4 s^{2} 4 p^{2}$ and $3 s^{2} 3 p^{5} 3 d^{5} 4 s^{2} 4 f$. The CSFs generated from these configurations were added to the list of reduced CSFs in step (a) for each of the symmetries. The relativistic effects are included via the Breit-Pauli approximation: the operators used are the mass correction and Darwin terms, plus a modified spinorbit interaction which reproduces almost exactly the values of the matrix elements of the full spin-orbit plus spin-other-orbit operators. We then used these sets of CSFs and the above listed Breit-Pauli operators to create the LSJ Hamiltonian matrix, with all possible LS symmetries combined for each of the even $J$ values (0.5-6.5). The total number of CSFs used for each of the $J$ values are follows:

\begin{tabular}{llllllll}
\hline \hline$J$ & 0.5 & 1.5 & 2.5 & 3.5 & 4.5 & 5.5 & 6.5 \\
\hline CSFs & 8339 & 12915 & 16145 & 15313 & 11589 & 6024 & 2167 \\
\hline
\end{tabular}

The Hamiltonian matrix for each of the $J$ values was then diagonalised to obtain the ab initio energies and eigenvectors for the required levels. The excitation energies of these levels relative to the ground state, $3 \mathrm{~d}^{6}\left({ }^{5} \mathrm{D}\right) 4 \mathrm{~s}^{6} \mathrm{D}_{4.5}$, are in general higher than the corresponding experimental energies (Ralchenko et al. 2008) by between a few tens even up to a few thousand $\mathrm{cm}^{-1}$. Using our fine-tuning technique whereby we make adjustments to the diagonal elements of the Hamiltonian matrix, we were able to bring our calculated energies very close to those of experimental energies. These adjustments to the Hamiltonian matrix give rise to the corresponding changes to the eigenvector compositions.

In the present calculation we have carefully studied the level mixing of the two neighbouring levels of $a^{4} \mathrm{G}_{5.5}$ and $b^{2} \mathrm{H}_{5.5}$ separated by $741.397 \mathrm{~cm}^{-1}$ and arranged them in the format $\mathrm{M}(\mathrm{N})$ where $\mathrm{M}$ is the percentage composition of the leading level and $\mathrm{N}$ is the next leading percentage composition as shown in Table 1.

It may be seen that the ab initio value of the crucial energy separation of the strongly interacting levels is in error by only $47 \mathrm{~cm}^{-1}$ and the fine-tuned separation is almost exact. The calculations " $A$ " and "B" are produced by changing only the finetuning correction of the $b^{2} \mathrm{H}_{5.5}$ level, keeping the $a^{4} \mathrm{G}_{5.5}$ level energy unchanged. Our aim in undertaking these calculations is simply to show that a relatively small change in the calculated energy separation of the two levels leads to substantial changes in the mixing of the two levels. It is clear that as the energy gap between the two levels increases, both the levels become purer and mixing between them becomes weaker.

\section{Results}

In the present research note our primary aim is to consider the ratio of the lifetimes of these two levels. The SUPERSTRUCTURE (SST) and Cowan (HFR) calculations of Quinet et al. (1996) predicted a lifetime ratio of between 8.5 and 7.4 respectively, with Garstang (1962) giving almost 12, whereas the experimental ratio is only 5 . We present in Table 2 all the transition probabilities contributing to the lifetimes of $b^{2} \mathrm{H}_{5.5}$, with corresponding results for $a^{4} \mathrm{G}_{5.5}$ displayed in Table 3, for the three cited calculations as well as our own fine-tuned results. We also show some results of the "A" and "B" calculations, but only for the most significant transitions. It is interesting to note
Table 1. Mixing coefficients of two neighbouring levels $a^{4} \mathrm{G}_{5.5}$ and $b^{2} \mathrm{H}_{5.5}$ in four different calculations.

\begin{tabular}{cccc}
\hline \hline Calculation & $a^{4} \mathrm{G}_{5.5}\left(b^{2} \mathrm{H}_{5.5}\right)$ & $b^{2} \mathrm{H}_{5.5}\left(a^{4} \mathrm{G}_{5.5}\right)$ & $\mathrm{EG}\left(\mathrm{cm}^{-1}\right)$ \\
\hline Fine-tuned & $76(17)$ & $75(17)$ & 745.270 \\
ab initio & $80(14)$ & $80(14)$ & 792.457 \\
A & $82(12)$ & $81(12)$ & 870.261 \\
B & $84(10)$ & $83(10)$ & 931.815 \\
\hline
\end{tabular}

Notes. The NIST energy gap (EG) is $741.397 \mathrm{~cm}^{-1}$ (Ralchenko et al. 2008).

from Table 2 that the HFR results of Quinet et al. (1996) agree quite closely with our ab initio calculations. For the strongest of the transitions, those involving $a^{4} \mathrm{~F}$, the SST results are lower than the HFR results, although Quinet et al. recommend the SST results. We note though that the fine-tuning process substantially changes the $A$-values, because of the increased component of the ${ }^{4} \mathrm{G}$ in the ${ }^{2} \mathrm{H}$ wave function even though, as shown in Table 1 , the corrections are quite small. We note that our calculation A (12\% mixing) still gives values similar to the ab initio results, while calculation B (10\% mixing) leads to values approaching the SST result of Quinet et al. (1996). A similar pattern emerges for the $a^{4} \mathrm{~F}_{3.5} \rightarrow b^{2} \mathrm{H}_{5.5}$ transition. This indicates that the level mixings between $a^{4} \mathrm{G}_{5.5}$ and $b^{2} H_{5.5}$ in the SST and HFR calculations of Quinet et al. (1996) were possibly somewhat similar to those of our ab initio, or even to the " $\mathrm{A}$ " and "B", calculations. Two other significant transitions in this Table involve $a^{2} \mathrm{G}_{4.5}$ and $a^{4} \mathrm{H}_{4.5}$, and also show a similar equivalence of our ab initio results and HFR results of Quinet et al. (1996). However, our fine tuned results for the former are about $27 \%$ lower and for the latter about $50 \%$ higher than corresponding HFR results.

The corresponding results for the transitions with $a^{4} \mathrm{G}_{5.5}$ as the upper level are shown in Table 3. This time, our $A$-values are much lower than those of previous authors. Again these are affected by variation in the mixing between the two upper levels, though proportionally not as much as for the transitions in Table 2, essentially because the ${ }^{4} \mathrm{G}$ component is already at $76 \%$ in our calculation. Clearly, as that component increases, with corresponding decrease in the mixing, as in calculations " $\mathrm{A}$ " or "B", the $A$-value also slowly increases.

In Table 4 we compare our calculated lifetimes of a number of levels with values obtained by others. We have selected levels for which experimental measurements have been obtained by the same group, based at Lund, but at various times. We note firstly that our value of $3.7 \mathrm{~s}$ for the lifetime of $b^{2} \mathrm{H}_{5.5}$ is very close to the experimental value of $3.8 \mathrm{~s}$ and is the only calculated result which lies inside the experimental error bars. By contrast, our result for $a^{4} \mathrm{G}_{5.5}$ lies outside the rather wider experimental error bars. We discuss this further in Sect. 4.3.

\section{Discussion}

In view of the disparity amongst the calculations, and between them and experiment, it is desirable that some critical discussion of accuracy is undertaken. Let us begin with our own work.

\subsection{The present calculations}

For the M1 transitions shown in Tables 2 and 3, there is quite close agreement amongst the calculations. However, the main contributions to the $J=5.5$ lifetimes are E2 transitions, principally those with $a^{4} \mathrm{~F}_{\mathrm{J}}$ as the lower level. For this discussion we 
N. C. Deb and A. Hibbert: Accurate $[\mathrm{Fe} \mathrm{II}]$ transition rates $(R N)$

Table 2. Dominant $A$-values (in s ${ }^{-1}$ ) contributing to the lifetime calculation of $3 \mathrm{~d}^{6}\left({ }^{3} \mathrm{H}\right) 4 \mathrm{~s} b^{2} \mathrm{H}_{5.5}$.

\begin{tabular}{|c|c|c|c|c|c|c|c|c|c|}
\hline Lower cfg. & ${ }^{2 S+1} L_{J}$ & Type & Garstang & $\begin{array}{c}\text { Quinet } \\
\text { SST }\end{array}$ & $\begin{array}{c}\text { Quinet } \\
\text { HFR }\end{array}$ & $\begin{array}{c}\text { Present } \\
\text { fine tuned }\end{array}$ & $\begin{array}{c}\text { Present } \\
\text { ab initio }\end{array}$ & $\begin{array}{c}\text { Present } \\
\text { A }\end{array}$ & $\begin{array}{c}\text { Present } \\
\text { B }\end{array}$ \\
\hline \multirow[t]{6}{*}{$3 d^{7}$} & ${ }^{4} \mathrm{~F}_{4.5}$ & E2 & 0.0450 & 0.0780 & 0.1030 & 0.1610 & 0.1058 & 0.1040 & 0.0878 \\
\hline & ${ }^{4} \mathrm{~F}_{3.5}$ & E2 & 0.0240 & 0.0337 & 0.0444 & 0.0593 & 0.0443 & 0.0413 & 0.0364 \\
\hline & ${ }^{2} \mathrm{G}_{4.5}$ & $\mathrm{E} 2, \mathrm{M} 1$ & 0.0135 & 0.0207 & 0.0216 & 0.0158 & 0.0209 & 0.0164 & 0.0169 \\
\hline & ${ }^{2} \mathrm{G}_{3.5}$ & E2 & 0.0003 & 0.0012 & 0.0012 & 0.0009 & & & \\
\hline & ${ }^{2} \mathrm{H}_{5.5}$ & E2 & 0.0026 & 0.0043 & 0.0048 & 0.0033 & & & \\
\hline & ${ }^{2} \mathrm{H}_{4.5}$ & E2 & 0.0002 & 0.0000 & 0.0000 & 0.0002 & & & \\
\hline \multirow[t]{3}{*}{$3 \mathrm{~d}^{6}\left({ }^{3} \mathrm{H}\right) 4 \mathrm{~s}$} & ${ }^{4} \mathrm{H}_{6.5}$ & M1 & 0.0096 & 0.0101 & 0.0118 & 0.0179 & 0.0126 & 0.0137 & 0.0121 \\
\hline & ${ }^{4} \mathrm{H}_{5.5}$ & M1 & 0.0008 & 0.0000 & 0.0000 & 0.0032 & & & \\
\hline & ${ }^{4} \mathrm{H}_{4.5}$ & M1 & 0.0013 & 0.0013 & 0.0014 & 0.0012 & & & \\
\hline $3 d^{6}\left({ }^{3} F 2\right) 4 s$ & ${ }^{4} \mathrm{~F}_{4.5}$ & M1 & 0.0013 & 0.0014 & 0.0020 & 0.0052 & & & \\
\hline \multirow[t]{2}{*}{$3 d^{6}\left({ }^{3} G\right) 4 s$} & ${ }^{4} \mathrm{G}_{5.5}$ & M1 & 0.0000 & 0.0010 & 0.0020 & 0.0020 & & & \\
\hline & ${ }^{4} \mathrm{G}_{4.5}$ & M1 & 0.0000 & 0.0000 & 0.0000 & 0.0002 & & & \\
\hline Total $A$-value & & & 0.0986 & 0.1517 & 0.1922 & 0.2702 & & & \\
\hline Lifetime $(\tau)$ & & & $10.14 \mathrm{~s}$ & $6.592 \mathrm{~s}$ & $5.203 \mathrm{~s}$ & $3.7009 \mathrm{~s}$ & & & \\
\hline
\end{tabular}

Notes. Transitions from various levels to the $b^{2} \mathrm{H}_{5.5}$ level are arranged in ascending order of their energy positions.

Table 3. Dominant $A$-values (in s ${ }^{-1}$ ) contributing to the lifetime calculation of $3 \mathrm{~d}^{6}\left({ }^{3} \mathrm{G}\right) 4 \mathrm{~s}^{4} \mathrm{G}_{5.5}$.

\begin{tabular}{cccccccccc}
\hline \hline Lower cfg. & ${ }^{2 S+1} \mathrm{~L}_{\mathrm{J}}$ & Type & Garstang & $\begin{array}{c}\text { Quinet } \\
\text { SST }\end{array}$ & $\begin{array}{c}\text { Quinet } \\
\text { HFR }\end{array}$ & $\begin{array}{c}\text { Present } \\
\text { fine tuned }\end{array}$ & $\begin{array}{c}\text { Present } \\
\text { ab initio }\end{array}$ & $\begin{array}{c}\text { Present } \\
\text { A }\end{array}$ & $\begin{array}{c}\text { Present } \\
\text { B }\end{array}$ \\
\hline $3 \mathrm{~d}^{7}$ & ${ }^{4} \mathrm{~F}_{4.5}$ & E2 & 0.9000 & 1.0200 & 1.1200 & 0.8061 & 0.8223 & 0.8605 & 0.8790 \\
& ${ }^{4} \mathrm{~F}_{3.5}$ & E2 & 0.2100 & 0.2270 & 0.2500 & 0.1820 & 0.1879 & 0.1990 & 0.2046 \\
$3 \mathrm{~d}^{6}\left({ }^{5} \mathrm{D}\right) 4 \mathrm{~s}$ & ${ }^{4} \mathrm{D}_{3.5}$ & $\mathrm{E} 2$ & 0.0000 & 0.0000 & 0.0000 & 0.0005 & & & \\
$3 \mathrm{~d}^{7}$ & ${ }^{2} \mathrm{G}_{4.5}$ & $\mathrm{E} 2$ & 0.0004 & 0.0000 & 0.0000 & 0.0018 & & & \\
& ${ }^{2} \mathrm{H}_{5.5}$ & $\mathrm{E} 2, \mathrm{M} 1$ & 0.0000 & 0.0000 & 0.0000 & 0.0005 & & & \\
$3 \mathrm{~d}^{6}\left({ }^{3} \mathrm{H}\right) 4 \mathrm{~s}$ & ${ }^{4} \mathrm{H}_{6.5}$ & M1 & 0.0290 & 0.0211 & 0.0210 & 0.0219 & 0.0200 & 0.0243 & 0.0253 \\
& ${ }^{4} \mathrm{H}_{5.5}$ & $\mathrm{M} 1$ & 0.0150 & 0.0114 & 0.0117 & 0.0126 & & & \\
$3 \mathrm{~d}^{6}\left({ }^{3} \mathrm{~F} 2\right) 4 \mathrm{~s}$ & ${ }^{4} \mathrm{~F}_{4.5}$ & $\mathrm{M} 1$ & 0.0190 & 0.0132 & 0.0132 & 0.0156 & 0.0093 & 0.0100 & 0.0101 \\
\hline Total $A$-value & & & 1.1734 & 1.2927 & 1.4159 & 1.0415 & & & \\
\hline Lifetime $(\tau)$ & & & $0.852 \mathrm{~s}$ & $0.774 \mathrm{~s}$ & $0.706 \mathrm{~s}$ & $0.960 \mathrm{~s}$ & & & \\
\hline
\end{tabular}

Notes. Transitions from various levels to the $a^{4} \mathrm{G}_{5.5}$ level are arranged in ascending order of their energy positions.

Table 4. Lifetimes $\tau(\mathrm{s})$ of six metastable levels.

\begin{tabular}{lllcccc}
\hline \hline Configuration & Term & Experiment & Garstang & $\begin{array}{c}\text { Quinet } \\
\text { SST }\end{array}$ & $\begin{array}{c}\text { Quinet } \\
\text { HFR }\end{array}$ & $\begin{array}{c}\text { Present } \\
\text { fine tuned }\end{array}$ \\
\hline $3 \mathrm{~d}^{5} 4 \mathrm{~s}^{2}$ & $a^{6} \mathrm{~S}_{2.5}$ & $0.23(3)^{p}$ & 0.326 & 0.262 & 0.220 & 0.233 \\
$3 \mathrm{~d}^{6}\left({ }^{3} \mathrm{G}\right) 4 \mathrm{~s}$ & $a^{4} \mathrm{G}_{5.5}$ & $0.75(10)^{q}$ & 0.852 & 0.774 & 0.704 & 0.958 \\
$3 \mathrm{~d}^{6}\left({ }^{3} \mathrm{G}\right) 4 \mathrm{~s}$ & $a^{4} \mathrm{G}_{4.5}$ & $0.65(2)^{r}$ & 0.856 & 0.755 & 0.694 & 0.870 \\
$3 \mathrm{~d}^{6}\left({ }^{3} \mathrm{H}\right) 4 \mathrm{~s}$ & $b^{2} \mathrm{H}_{5.5}$ & $3.8(3)^{r}$ & 10.1 & 6.59 & 5.20 & 3.701 \\
$3 \mathrm{~d}^{6}\left({ }^{3} \mathrm{D}\right) 4 \mathrm{~s}$ & $b^{4} \mathrm{D}_{0.5}$ & $0.54(3)^{q}$ & 0.677 & 0.630 & 0.550 & 0.626 \\
$3 \mathrm{~d}^{6}\left({ }^{3} \mathrm{D}\right) 4 \mathrm{~s}$ & $b^{4} \mathrm{D}_{3.5}$ & $0.53(3)^{p}$ & 0.618 & 0.567 & 0.500 & 0.577 \\
\hline$\tau_{R}$ & & 5.07 & 11.9 & 8.51 & 7.39 & 3.86 \\
\hline
\end{tabular}

Notes. Experimental uncertainties are in brackets. $\tau_{R}$ is the ratio of the lifetime of $b^{2} \mathrm{H}_{5.5}$ to $a^{4} \mathrm{G}_{5.5}$. ${ }^{(p)}$ Rostohar et al. (2001), (q) Gurell et al. (2009), ${ }^{(r)}$ Hartman et al. (2003).

will focus on the case of $J=4.5$. There are several potential sources of error.

Firstly, we remark that while the principal mixing is between the upper levels $-3 \mathrm{~d}^{6} 4 \mathrm{~s}^{4} \mathrm{G}_{5.5}$ and $3 \mathrm{~d}^{6} 4 \mathrm{~s}^{2} \mathrm{H}_{5.5}$ - some other nonnegligible mixing does arise, in the sense of contributing significantly to the $A$-values of the two transitions under discussion. Specifically, $3 \mathrm{~d}^{7}{ }^{4} \mathrm{~F}_{4.5}$ mixes with $3 \mathrm{~d}^{6} 4 \mathrm{~s}^{2} \mathrm{G}_{4.5}$ and the two upper states mix also with $3 \mathrm{~d}^{7}{ }^{2} \mathrm{H}_{5.5}$ and $3 \mathrm{~d}^{6} 4 \mathrm{~s}^{4} \mathrm{H}_{5.5}$. We find that the optimal 4 s function is almost independent of the LS term, but the $3 \mathrm{~d}$ function changes significantly depending on the occupancy of the $3 \mathrm{~d}$ subshell. That is, the $3 \mathrm{~d}$ function of $3 \mathrm{~d}^{7}$ states, while being similar from one to another, is quite different from that of the $3 d^{6} 4 s$ states, and different again from that of the $3 d^{5} 4 s^{2}$ states. In addition to the core orbitals of $1 \mathrm{~s}, 2 \mathrm{~s}, 2 \mathrm{p}, 3 \mathrm{~s}$ and $3 \mathrm{p}$, our calculations have included four d-functions to allow for this variation, and four s-functions to describe any variation in the $4 \mathrm{~s}$ function from state to state. However, a more accurate set of results might be obtained if we had used 'non-orthogonal' orbitals, in which particularly the different $3 \mathrm{~d}$ functions were included explicitly in the calculations.

Secondly, our calculations have involved rather extensive CI using these orbitals as well as four additional p-functions plus 
4f, and allowing all single and double replacements from the $3 \mathrm{~d}$ and $4 s$ subshells in each of $3 d^{7}, 3 d^{6} 4 s$ and $3 d^{5} 4 s^{2}$ to any of these orbitals. Furthermore, as noted in Sect. 2, we include the main correlation effects in the $3 \mathrm{~s}$ and $3 \mathrm{p}$ subshells. In this way, we account for the term-dependency of the valence electrons and a substantial amount of electron correlation in all the states under investigation. This represents by far the most extensive ab initio treatment of electron correlation undertaken to date. We believe that the source of error noted in the previous paragraph will be largely overcome by the extensive nature of this calculation. In addition, final refinements using our "fine-tuning" process ensures good agreement between our calculated energies and the corresponding experimental values. But this again is a potential source of error. While experience in the past has led us to believe that this process is capable of replicating closely the effect of the addition of (many) more CSFs, it is nonetheless not quite as accurate as actually adding in those CSFs. To check this, we have undertaken some very simple calculations, involving only a few CSFs, followed by fine-tuning, and we found that the level of CI mixing between the important terms was quite close to the large-scale calculations. So we think that this potential source of error is also small.

Thirdly, we have represented relativistic effects by using the Breit-Pauli operators. This is in itself a further approximation and therefore source of error, but for Fe II, this approximation should again have only a very small influence on the results. Much more significant is, we believe, our omission of the spinspin term and particularly the replacement of the spin-other-orbit term by a modified spin-orbit term. The parameters were chosen so that this modified operator gave almost identical matrix elements between key CSFs to those of the full spin-orbit and spinother-orbit operators combined. And in a calculation actually including all the operators instead, but with rather fewer CSFs, we found that the calculated energies differed by less than $15 \mathrm{~cm}^{-1}$ for the $J=4.5$ levels and $25 \mathrm{~cm}^{-1}$ for the $J=5.5$ levels. This must be seen in the context of correlation energies obtained in our calculation of the order of $40000 \mathrm{~cm}^{-1}$. But the problem we found is that the off-diagonal Hamiltonian matrix element between $3 \mathrm{~d}^{6} 4 \mathrm{~s}^{4} \mathrm{G}_{5.5}$ and $3 \mathrm{~d}^{6} 4 \mathrm{~s}^{2} \mathrm{H}_{5.5}$ reduced by about $15 \mathrm{~cm}^{-1}$, which is about $6 \%$ of its value, and so the mixing coefficient is reduced by this amount too. This suggests that the $A$-value of the transition between $a^{4} \mathrm{~F}_{4.5}$ and $b^{2} \mathrm{H}_{5.5}$ may be a little too high, even by up to $15 \%$ (because of the contributions from other LSJ symmetries), and the $A$-value of the other transition may be too low by $4-5 \%$.

Fourthly, the overall magnitude of the $A$-values is governed by the square of the radial quadrupole integrals $\left\langle P_{\mathrm{nl}}\left|r^{2}\right| P_{n^{\prime} l^{\prime}}\right\rangle$. The integral involved in the dominant contributions to the $A$ values has $n l=3 \mathrm{~d}$ and $n^{\prime} l^{\prime}=4 \mathrm{~s}$. It is therefore natural that the $3 \mathrm{~d}$ should be that of the $3 \mathrm{~d}^{7}{ }^{4} \mathrm{~F}_{4.5}$ and that happens to be the choice we made for the $3 \mathrm{~d}$ function. It is also the choice made by Garstang (1962) and our radial integrals agree very closely with his. Smaller contributions to the $A$-values involve $n^{\prime} l^{\prime}=3 \mathrm{~d}$, and for these, our choice of $3 \mathrm{~d}$ is not the most appropriate. But we believe that our additional d-functions make adequate allowance for this, and in any case those contributions are fairly small.

\subsection{Other calculations}

In the early work by Garstang (1962), Hamiltonian matrix elements were expressed in terms of a number of electrostatic and spin-orbit parameters, separately for each of $3 d^{7}, 3 d^{6} 4 s$ and $3 \mathrm{~d}^{5} 4 \mathrm{~s}^{2}$, together with other correction factors, and CI between these configurations was incorporated. In calculating the $A$ values, the radial integrals chosen depended on which $3 \mathrm{~d}$ function was the most relevant to each transition. Thus, for the two transitions discussed here, he used the $3 d$ function of the $3 d^{7}$ states, as we also have done. The main difference between the outcomes of our calculation and his is the calculated energy separations of the two upper states. Garstang obtained a difference of $1128 \mathrm{~cm}^{-1}$. This is considerably larger than the experimental value or even our ab initio value, shown in Table 1 . Therefore the mixing of the two upper levels in Garstang's calculation will be much weaker than in ours and leads to the much smaller $A$-value shown in the first line of Table 2. The first transition in Table 3 is consequentially larger than our value, but the sum of the two $A$-values (just less than $1.0 \mathrm{~s}^{-1}$ ) is similar in the two calculations, as might be expected given the use of the same $3 \mathrm{~d}$ function in the quadrupole radial integral.

The sum of the two $A$-values for the HFR calculation of Quinet et al. (1996) is rather larger, at $1.2 \mathrm{~s}^{-1}$, with a substantially larger value for the $A$-value of the $a^{4} \mathrm{~F}-a^{4} \mathrm{G}$ transition, when compared with our result, and a lower value for $a^{4} \mathrm{~F}-b^{2} \mathrm{H}$. In this calculation, the electrostatic radial integrals are used as parameters fitted to experimental energies, with (as in Garstang's work) different values for each of $3 d^{7}, 3 d^{6} 4 s$ and $3 d^{5} 4 s^{2}$. These fitted values are $15-20 \%$ lower than ab initio HF values, as is common in such calculations, but it is not clear if a similar scaling is applied to the quadrupole radial integral. Certainly, their value is consistent with a rather larger value for the quadrupole radial integral than we have obtained.

The SST method is closer in concept to our own, with substantial CI and the use of term energy corrections (TEC) which is akin to our fine-tuning process. The SST results of Quinet et al. (1996) lie below their HFR values for both transitions, but the low $A$-value of $a^{4} \mathrm{~F}-b^{2} \mathrm{H}$ suggests a much weaker mixing of the upper levels than we obtain. We note that the TECs for the two upper states are rather different, suggesting that their ab initio energies for these two levels are much further apart $\left(1148 \mathrm{~cm}^{-1}\right.$, close to Garstang's value) than our ab initio values or the experimental difference (see Table 1). The much more extensive CI in our calculation is a factor in our reaching such an improvement in the energy separation of the two upper states. Quinet et al. do include three d-functions in their calculation, though the scaling parameters of the model potentials used to obtain these functions are optimised so as to minimise the sum of 24 term energies, so inevitably some compromise is introduced, perhaps also in the size of the quadrupole radial integral. We remark that in our calculation, a very simple but fined-tuned CI did give an $A$-value of around $0.1 \mathrm{~s}^{-1}$ for the $a^{4} \mathrm{~F}_{4.5}-b^{2} \mathrm{H}_{5} .5$ transition but the additional CSFs introduced to account for the bulk of electron correlation omitted in the simple calculation each contributed small amounts so that the $A$-value was raised to $0.16 \mathrm{~s}^{-1}$.

\subsection{Comparison with experimental results}

The experimental values of the lifetimes of the upper levels (Gurell et al. 2009; Hartman et al. 2003), shown in Table 4, suggest $A$-values of around $1.0 \mathrm{~s}^{-1}$ for $a^{4} \mathrm{~F}-a^{4} \mathrm{G}$ and around $0.15 \mathrm{~s}^{-1}$ for $a^{4} \mathrm{~F}-b^{2} \mathrm{H}$. The latter is in keeping with our result, but the former is around $25 \%$ larger. However, we note that the error bars for the lifetime of $a^{4} \mathrm{G}$ are quite broad and the upper edge of the bar would correspond to an $A$-value of around $0.9 \mathrm{~s}^{-1}$.

We have included in Table 4 a comparison of our results with other work for several levels not discussed above. There is general agreement between the relatively recent determinations of 
the lifetime of the $a^{6} \mathrm{~S}_{2.5}$ level and some consensus for the $b$ ${ }^{4} \mathrm{D}_{3.5}$ level. However, for the other two levels included in Table 4 $-a^{4} \mathrm{G}_{4.5}$ and $b{ }^{4} \mathrm{D}_{0.5}$ - the HFR calculations of Quinet et al. (1996) are clearly the closest to the experimental determinations. We would remark that the SST results of Quinet et al. (1996) might be expected to be closer to ours than are the HFR values, since the SST method involves modelling only at the stage of determining the orbital radial functions (the radial integrals are not then scaled further). We are therefore surprised that it is the HFR results which give the best agreement with experiment, for these levels. Indeed, Quinet et al. (1996) state a preference for their SST results, a view supported by their "Note added in proof" which suggests that the HFR results may not be sufficiently converged with respect to the addition of further configurations.

Another point of discrepancy in Table 4 concerns the $J$-dependence of the lifetimes of levels within a term. The $a^{4} \mathrm{G}_{5.5}$ and $a^{4} \mathrm{G}_{4.5}$ lifetimes differ in our calculations by $0.088 \mathrm{~s}$, close to the experimental difference of 0.10 , whereas in the other calculations no substantial difference is noted. By contrast, for the $b$ ${ }^{4} \mathrm{D}$ levels, all the calculations predict a difference of $0.05-0.06 \mathrm{~s}$ between $J=0.5$ and $J=3.5$, whereas the experimental difference is much smaller.

\section{Conclusion}

The present work is by far the most extensive calculation of these transitions so far undertaken. Ab initio energy differences are in much better agreement than had previously been achievable so that that fine-tuning process, particularly for the transitions discussed here, made only a small difference to the ab initio $A$-values. Still, the degree of mixing between $a^{4} \mathrm{G}_{5.5}$ and $b^{2} \mathrm{H}_{5.5}$ remains very sensitive to quite small potential errors, such as in the Hamiltonian matrix elements between them. Our calculated lifetime for $b^{2} \mathrm{H}_{5.5}$ agrees well with experiment, while the lifetime comparison for $a^{4} \mathrm{G}_{5.5}$ is made more difficult because of experimental uncertainties, though we do not expect that our calculated $A$-values for the strongest transitions are more than a few percent below their true values. That said, it is clear from the discussion of Sect. 4.3 that some unresolved issues remain. It would be useful to undertake a calculation as extensive as the present one, but using "non-orthogonal" orbitals, thus allowing for the $3 \mathrm{~d}$ functions to be optimal for each of the states under investigation.

Acknowledgements. The authors would like to thank PPARC/STFC, UK for support under Rolling Grant PP/D00103X/1.

\section{References}

Clementi, E., \& Roetti, C. 1974, At. Data Nucl. Data Tables, 14, 177 Corrégé, G., \& Hibbert, A. 2005, ApJ, 627, L157

Corrégé, G., \& Hibbert, A. 2006, ApJ, 636, 1166

Garstang, R. H. 1962, MNRAS, 124, 321

Gull, T. R., Ishibashi, K, Davidson, K., \& the Cycle 7 STIS Go Team 1999, in Eta Carinae at The Millennium, ASP Conf. Ser., 179, 144

Gurell, J., Hartman, H., Blackwell-Whitehead, R., et al. 2009, A\&A, 508, 525

Hartman, H., Derkatch, A., Donnelly, M.P., et al. 2003, A\&A, 397, 1143

Hibbert, A. 1975, Comput. Phys. Commun., 9, 141

Hibbert, A., Glass, R., \& Fischer, C. F. 1991, Comput. Phys. Commun., 64, 455 Merrill, P. W. 1928, ApJ, 67, 391

Palmeri, P., Quinet, P., Biémont, E., et al. 2008, J. Phys. B, 41, 125703

Quinet, P., Le Dourneuf, M., \& Zeippen, C. J. 1996, A\&AS, 120361

Ralchenko, Yu., Kramida, A. E., Reader, J., et al. 2008, NIST Atomic Spectra Database, version 3.1.5, http://physics.nist.gov/asd3, National Institute of Standards and Technology, Gaithersburg, MD

Rostohar, D., Derkatch, A., Hartman, H., et al. 2001, Phys. Rev. Lett., 86, 1446 Royen, P., Gurell, J., Lundin, P., et al. 2007, Phys. Rev. A, 76, 030502(R) 